\title{
Simulating Collider Physics on Quantum Computers Using Effective Field Theories
}

\author{
Christian W. Bauer $\odot^{*}$ and Benjamin Nachman $\oplus^{\dagger}$ \\ Physics Division, Lawrence Berkeley National Laboratory, Berkeley, California 94720, USA \\ Marat Freytsis \\ NHETC, Department of Physics and Astronomy, Rutgers University, Piscataway, New Jersey 08854, USA \\ and Physics Division, Lawrence Berkeley National Laboratory, Berkeley, California 94720, USA
}

(Received 18 May 2021; accepted 12 October 2021; published 18 November 2021)

\begin{abstract}
Simulating the full dynamics of a quantum field theory over a wide range of energies requires exceptionally large quantum computing resources. Yet for many observables in particle physics, perturbative techniques are sufficient to accurately model all but a constrained range of energies within the validity of the theory. We demonstrate that effective field theories (EFTs) provide an efficient mechanism to separate the high energy dynamics that is easily calculated by traditional perturbation theory from the dynamics at low energy and show how quantum algorithms can be used to simulate the dynamics of the low energy EFT from first principles. As an explicit example we calculate the expectation values of vacuum-to-vacuum and vacuum-to-one-particle transitions in the presence of a time-ordered product of two Wilson lines in scalar field theory, an object closely related to those arising in EFTs of the standard model of particle physics. Calculations are performed using simulations of a quantum computer as well as measurements using the IBMQ Manhattan machine.
\end{abstract}

DOI: 10.1103/PhysRevLett.127.212001

It is well known that quantum computers can in principle simulate the time evolution of quantum field theories [1]. The main technique involves disretizing the spatial degrees of freedom by introducing a lattice [2-4], and digitizing the field values at a given lattice point [5-13]. This turns the uncountably infinite dimensional Hilbert space of standard quantum field theories into a finite dimensional Hilbert space of dimension

$$
n_{H}=n_{\phi}^{\left(N^{d}\right)},
$$

where $n_{\phi}$ denotes the dimensionality of the Hilbert space for a given lattice point, $N$ is the total number of lattice points in each spatial direction, and $d$ represents the number of spatial dimensions. The physical volume of the lattice is determined by the distance between adjacent lattice points $\delta x$ in each direction and is given by

$$
V=(N \delta x)^{d} \equiv L^{d}
$$

The total number of qubits required for such a simulation is given by

Published by the American Physical Society under the terms of the Creative Commons Attribution 4.0 International license. Further distribution of this work must maintain attribution to the author(s) and the published article's title, journal citation, and DOI. Funded by SCOAP ${ }^{3}$.

$$
n_{Q}=\mathcal{O}\left(\log _{2} n_{H}\right)=\mathcal{O}\left(N^{d} \log _{2} n_{\phi}\right) .
$$

The discretization and finite volume of space introduce upper and lower cutoffs to the energies $E$ over which the resulting lattice field theory is a good approximation for the continuum. In particular, one finds

$$
\frac{1}{N \delta x} \lesssim E \lesssim \frac{1}{\delta x}
$$

which implies that the range of energies that can be described is directly proportional to the number of lattice sites per dimension. In principle, to have access to the full dynamics of the Large Hadron Collinder (LHC), one would need to describe the energy range between $\mathcal{O}(10 \mathrm{MeV})$ (the smallest resolvable transverse momentum between hadrons) and $7 \mathrm{TeV}$ (the beam energy of the LHC). To fully capture this energy range would require a lattice with $\mathcal{O}\left(10^{6}\right)$ lattice points in each dimension, and more than $10^{18}$ qubits to reproduce the resulting physical system. Even if one does not require the full energy range up to the LHC center-of-mass energy, the number of qubits required for a full simulation will clearly remain beyond the realm of feasibility for a long time to come.

For many observables of interest at the LHC, physics at short distances is reliably computed in fixed order perturbation theory, and high precision can be reached with existing techniques. Physics at lower energies introduces new significant challenges. Asymptotic freedom [14,15] 
implies that the strong coupling constant becomes large at low energies, increasing the production of additional particles. An energetic particle can thus easily radiate additional soft and collinear particles, resulting in a collimated jet of particles. This means that fixed order perturbative calculations are of little use to describe lower energy effects such as the precise makeup of jets, and other techniques such as resummation [16-18] and parton showers $[19,20]$ have to be used to make predictions. At even lower energies, the fully nonperturbative dynamics of hadronization dominate. While classical lattice methods provide spectral information about the strongly coupled limit of the theory, the dynamics of hadronization is currently only understood through phenomenological models and form factors extracted from data. While these techniques have been quite successful and it has even been shown that quantum algorithms can be used to include quantum interference effects in some models of parton showers [21], it would be a significant breakthrough were it possible to simulate these low energy dynamics from first principles.

In this Letter we show that this is indeed possible by using effective field theories (EFTs) which have been designed to reproduce the desired long distance physics. (For work on simulating EFTs not related to collider physics, see [22-27]). The dynamics of these EFTs can then be simulated directly and from first principles on a quantum computer. Since the energy range that needs to be simulated is much smaller than that of the full theory, the resource requirements are orders of magnitudes smaller than those required to simulate the full theory. Additionally, the EFT simplifies the description of the initial and final state and significantly reduces the resource requirements of their implementation. For example, consider an observable measured on two jets, each with an energy of $\mathcal{O}(1 \mathrm{TeV})$ and an invariant mass of $\mathcal{O}(50 \mathrm{GeV})$. Even restricting ourselves to observables insensitive to hadronization, a generic observable of the momenta of final state particles in the full theory requires a simulation of the range $1 \mathrm{GeV} \lesssim E \lesssim 2 \mathrm{TeV}$. As we discuss below, the EFT only needs to capture $1 \mathrm{GeV} \lesssim E \lesssim 50 \mathrm{GeV}$. The number of qubits required to simulate the EFT is smaller by a factor of $(2000 / 50)^{3} \approx 10^{5}$, and this factor increases rapidly as the range of the full theory is increased.

The EFT relevant for hadronic jet physics is softcollinear effective theory (SCET) [28-31]. Using this EFT, a typical cross section describing a physical observable at the LHC can be factorized into separate pieces [32-35]

$$
\sigma=H \otimes J_{1} \otimes \ldots \otimes J_{n} \otimes S .
$$

Here $H$ denotes a coefficient describing the short distance physics which can be computed reliably in standard perturbation theory, while $J_{i}$ and $S$ denote squares of matrix elements of operators in SCET. The jet function $J_{i}$ denotes physics arising from collinear degrees of freedom that all have small momentum relative to each other (while moving collectively with a large energy), with the different jet functions completely decoupled from one another. The soft function $S$ denotes physics arising from soft degrees of freedom, which all have small absolute momentum (in the frame of the collider). These matrix elements describe the transition of a simple initial state produced in the short distance interaction $H$ into a final state, with the dynamics described by the EFT Hamiltonian. The final states that arise can contain a large number of particles. State of the art techniques use operator renormalization to compute the overall scale dependence of the jet and soft functions, and then compute the relevant matrix elements perturbatively, such that the effects arising from the high multiplicity final states or hadronization cannot be properly included. Classical lattice techniques are also not suitable for the computation of matrix elements of SCET operators, since the long-distance dynamics is governed by massless modes which are inherently Minkowskian in nature. Having a simulation of the dynamics of SCET (or a different EFT for other problems) will allow for a full nonperturbative calculation of any matrix elements.

The EFT reproduces the full theory result up to power corrections, whose size depends on the kinematics of the process studied. For many observables of interest, these power corrections are considerably smaller than the perturbative corrections present in either the full theory or EFT matrix elements.

Since individual jet functions do not interact with one another, their dynamics can be simulated in the reference frame where all particles have small absolute momentum [36,37]. This requires simulating the dynamics of the original field theory (with any degrees of freedom that only contribute to short distance effects removed), but with a much smaller energy range required to be simulated. To achieve this on a quantum computer, one needs a setup very similar to that for the full theory, but reliable calculations can be achieved with much coarser lattices than those one would need for a simulation of the full theory.

In the soft function, on the other hand, the energetic particles no longer contribute to the dynamics of the theory. Instead, their effect is captured by a so-called Wilson line, which describes the interaction of a charge moving along a fixed world line with the bath of soft degrees of freedom. The physics underlying this observation is that soft particles cannot change the direction of energetic particles in a meaningful way. This implies that energetic particles can be included in the EFT as a static object (Wilson line) described by the relevant quantum numbers (charge, color, etc.) moving along a fixed world line along a lightlike direction. Matrix elements in the soft theory therefore need to compute the dynamics of a Hamiltonian describing the soft bath of particles in the presence of such Wilson lines. For gauge theories, such as those describing the three 
fundamental forces contained in the standard model, Wilson lines are given by path ordered exponentials of the relevant gauge fields [31]

$$
Y_{n}=P \exp \left[i g \int_{0}^{\infty} d s n \cdot A\left(x^{\mu}=n^{\mu} s\right)\right] .
$$

Here $A^{\mu}$ is the soft gauge field and $n^{\mu}$ describes the direction of the lightlike direction $n^{\mu}=(1, \mathbf{n})$ with $\mathbf{n}^{2}=$ 1 such that $n_{\mu} n^{\mu}=0$. Thus, the gauge field is evaluated on a path going from the origin to spatial infinity along the world line characterized by the direction $n^{\mu}$. The dynamics of the soft gauge field is described by its full theory Hamiltonian.

The soft function for a process containing two energetic particles of zero total charge moving back-to-back requires two Wilson lines, $Y_{n}$ for the particle moving in the $n$ direction and $Y_{\bar{n}}^{\dagger}$ for the antiparticle moving in the $\bar{n}^{\mu}=$ $(1,-\mathbf{n})$ direction. The matrix elements required in the soft sector are given by

$$
\left\langle X\left|T\left[Y_{n} Y_{\bar{n}}^{\dagger}\right]\right| \Omega\right\rangle,
$$

where $T$ denotes the time-ordering operator, $|\Omega\rangle$ denotes the ground state of a Hilbert space containing only the gauge degree of freedom, and $|X\rangle$ is the final state. If reproducing multiparticle weakly coupled final states, $|X\rangle$ will contain a fixed number of gauge momentum modes with given momenta $k_{i}^{\mu}$. To compute the soft function for a given observable one needs to sum over all possible final states that can contribute to this observable. Traditional perturbative calculations [38-44] can only compute these matrix elements for final states $|X\rangle$ containing a small number of particles and at low order in perturbation theory, since the complexity of the calculation increases factorially with the power of the coupling constant $g$. They therefore do not compute the full matrix element for a given observable, but only a perturbative approximation. For large coupling constants, which is the relevant case for quantum chromodynamics (QCD) at low energies, such perturbative calculations can give rise to large uncertainties.

Simulating the full dynamics of the field theory would provide the full nonperturbative result for the soft matrix element. To evaluate the matrix element on a quantum computer one first needs to define circuits that perform time evolution of the system, as well as circuits that can create and measure the ground and excited states of the theory. In addition, one needs to create circuits that can correctly interleave the implementation of nonlocal Wilson line operators with the evolution of the system to reproduce their time-ordered product.

In the following we discuss how to compute matrix elements of Wilson line operators $Y_{n}$ analogous to Eq. (7), but for a massless scalar, rather than gauge, theory. The EFT is insensitive to the precise origin of the Wilson lines, but a particularly straightforward realization would result from a pair of highly energetic fermions coupled to massless scalars through a Yukawa coupling. When constructing the explicit circuit we also limit ourselves to $(1+1)$ dimensions, mainly to restrict the quantum resources required such that it can be implemented on currently existing hardware. This allows us to omit some technical complications that arise when dealing with gauge theories (gauge transformations, the existence of unphysical polarizations, etc.), while capturing all the resulting simplification of working within an EFT.

To be precise, we consider a massless field theory in $(1+1)$ dimensions with Hamiltonian and Wilson lines defined by

$$
\begin{aligned}
H & =\int d x \frac{1}{2}\left(\dot{\phi}^{2}-\phi \partial^{2} \phi\right), \\
Y_{n} & =P \exp \left[i g \int_{0}^{\infty} d s \phi\left(x^{\mu}=n^{\mu} s\right)\right] .
\end{aligned}
$$

In the Supplemental Material [45], we discuss how working in $(1+1)$ dimensions gives rise to several effects not present in higher dimensions, and how these generalize to higher dimensions.

We discretize the position $x$ into an odd number of lattice points, labeling the positions by $x_{0}, \ldots, x_{N-1}$. To eliminate the zero-momentum mode of the theory, we impose twisted boundary conditions [46-49]. The result is a theory defined at discrete positions $x$ and momenta $p$ given by $x_{i}=x_{\min }+i \delta x$ and $p_{i}=p_{\min }+i \delta p$ with $x_{\min }=-(N-1) \delta x / 2, p_{\min }=$ $-\pi / \delta x$ and $\delta p=2 \pi / N \delta x$. Writing $\phi_{i} \equiv \phi\left(x_{i}\right)$, the twisted boundary conditions correspond to the condition $\phi_{i+N}=-\phi_{i}$. The Hamiltonian becomes [5]

$$
H=\frac{\delta x}{2} \sum_{i=0}^{N-1}\left[\dot{\phi}_{i}^{2}-\phi_{i}\left[\nabla^{2} \phi\right]_{j}\right],
$$

where the lattice operator $\nabla^{2}$ is defined through its action on a field as $\left[\nabla^{2} \phi\right]_{i}=\left(2 \phi_{i}-\phi_{i-1}-\phi_{i+1}\right) / \delta x^{2}$. Because of the twisted boundary conditions $\left[\nabla^{2} \phi\right]_{0}=\left(2 \phi_{0}+\phi_{N-1}-\right.$ $\left.\phi_{1}\right) / \delta x^{2}$ and $\left[\nabla^{2} \phi\right]_{N-1}=\left(2 \phi_{N-1}-\phi_{N-2}+\phi_{0}\right) / \delta x^{2}$. The Wilson line operators can be written as

$$
\begin{aligned}
& Y_{n}=P \exp \left[i g \delta x \sum_{i=n_{0}}^{2 n_{0}} \phi_{x_{i}}\left(t=x_{i}-n_{0}\right)\right], \\
& Y_{\bar{n}}^{\dagger}=P \exp \left[-i g \delta x \sum_{i=0}^{n_{0}} \phi_{x_{i}}\left(t=n_{0}-x_{i}\right)\right],
\end{aligned}
$$

where $n_{0}=(N-1) / 2$ denotes the point at the center of the lattice.

We represent the field theory through the field values at each lattice position, and in order to describe the theory on a digital quantum computer one needs to digitize the 
continuous field value at each lattice point [8]. Choosing $n_{Q}$ qubits per lattice site allows for $n_{\phi} \equiv 2^{n_{Q}}$ different field values. For each lattice point, the possible field values are chosen to be by $\phi_{i}^{(k)}=-\phi_{\max }+k \delta \phi$, with $\delta \phi=2 \phi_{\max } /\left(n_{\phi}-1\right)$. The value of $\phi_{\max }$ has to be chosen to optimize the digitized description, which for free fields is accomplished by

$$
\phi_{\max }=\frac{1}{\sqrt{\delta x \bar{\omega}}} \sqrt{\frac{\pi}{2} \frac{\left(n_{\phi}-1\right)^{2}}{n_{\phi}}},
$$

where

$$
\bar{\omega}=\frac{1}{N} \sum_{i} \omega_{i}, \quad \omega_{i}=\frac{2}{\delta x}\left|\sin \frac{p_{i} \delta x}{2}\right| .
$$

For $\bar{\omega}=1$, as is the case for a single lattice site with $\omega=1$, corresponding to a single harmonic oscillator, Eq. (11) reproduces the empirical numerical values obtained in [8].

To implement the Wilson line operator we first rewrite the time-ordered product of the two Wilson lines as

$$
\begin{aligned}
T\left[Y_{n} Y_{\bar{n}}^{\dagger}\right]= & e^{-i H n_{0} \delta x} \exp \left[i g \delta x\left(\phi_{x_{2 n_{0}}}-\phi_{x_{0}}\right)\right] \\
& \times e^{i H \delta x} \exp \left[i g \delta x\left(\phi_{x_{2 n_{0}-1}}-\phi_{x_{1}}\right)\right] \\
& \times \ldots \times e^{i H \delta x} \exp \left[i g \delta x\left(\phi_{x_{n_{0}}}-\phi_{x_{n_{0}}}\right)\right],
\end{aligned}
$$

where we have used the time translation operator to make the time dependence on the field operators explicit. Thus, the Wilson line operator consists of a sequence of timeevolution operators for a time interval corresponding to the lattice spacing and exponentials of the field operator. The last time evolution evolves the state back from the largest time to which the Wilson lines can be sensibly evolved, namely, $t_{\max }=n_{0} \delta x$, to $t=0$ at which all states are defined.

Ultimately, to make contact with the continuum field theory any such simulation will have to be performed on a series of increasing lattices, and the result extrapolated to the $N \rightarrow \infty, \delta x \rightarrow 0$ limit. Any parameters of the theory present in the continuum must be suitably matched for this procedure to yield meaningful results. For local terms in the Hamiltonian, this procedure is discussed in detail in [5]. Dealing with a massless theory simplifies this procedure since only local interactions (of which in the present case there are none) need to be matched. However, the EFT will also require the matching of Wilson line operators, which is complicated by their nonlocal nature and sensitivity to total lattice size, as discussed in the Supplemental Materials. In this Letter, we work at fixed lattice size and we leave the detailed investigation of these issues to future work.

The implementation of the exponential of the field operator, as well as the time evolution operator, follows the discussion in [8] and uses the fact that the digitized field $\phi_{i}^{(k)}$ can be written in terms of sums of $\sigma_{z}$ operators. This implies that the exponential of products of fields $\phi_{i}$ can be implemented through combinations of CNOT gates and $R_{Z}$ rotations [6-8]. The exponential of the conjugate operator $\dot{\phi}^{2}$ can be implemented by taking a quantum Fourier transformation of the exponential of the operator $\phi^{2}$. These can then be combined via the Suzuki-Trotter formula [50-52]. For details, see the Supplemental Materials. The initial ground state of the scalar field theory is a multivariate Gaussian distribution, which can be created using the approach of Kitaev and Webb (KW) [53]. To identify states of definite multiplicity and momentum in $|X\rangle$ one can follow the general ideas laid out in [1,5].

Our quantum circuit has been implemented in QISKIT [54] and is available from the authors upon request. In this first exploratory paper we compute the foundational quantities, namely,

$$
\mathcal{Y}_{X}=\left|\left\langle X\left|T\left[Y_{n} Y_{\bar{n}}^{\dagger}\right]\right| \Omega\right\rangle\right|^{2}
$$

for $|X\rangle=|\Omega\rangle$ and $|X\rangle=\left|p_{i}\right\rangle$, the one-particle momentum eigenstates of the theory. It should be noted that these quantities are not infrared (IR) safe, and will therefore depend on the IR scale in the problem, the lattice size $L$. However, as discussed in more detail in the Supplemental Materials, there is no nontrivial IR safe observable that can be defined in $(1+1)$ dimensions, and these transition rates are therefore representative quantities of what can be computed in this theory.

The quantum circuit for this measurement can be represented as

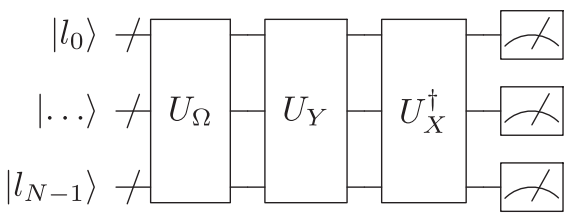

where $\left|l_{n}\right\rangle$ denotes the register of qubits for the $n$th lattice site. This creates the multivariate Gaussian vacuum state from the initial state with all qubits zero using $U_{\Omega}$, acts on this vacuum with the time ordered product of the two Wilson lines using $U_{Y}$, and finally applies the inverse of the state preparation of state $|X\rangle$. The details of these various circuits can be found in the Supplemental Material.

For our numerical results, we work with an $N=3$ site lattice with spacing $\delta x=1$. With only three lattice sites, the Wilson line operator simplifies to

$\mathcal{Y}_{X}=\left|\left\langle X\left|T\left[Y_{n} Y_{\bar{n}}^{\dagger}\right]\right| \Omega\right\rangle\right|^{2}=\left|\left\langle X\left|e^{i g \delta x\left(\phi_{x_{2}}-\phi_{x_{0}}\right)}\right| \Omega\right\rangle\right|^{2}$,

since all time evolution operators act on the initial or final eigenstate of the Hamiltonian only and can therefore be neglected as contributing an overall phase. In Fig. 1 we show the dependence of the expectation values $\mathcal{Y}_{X}$ on the 


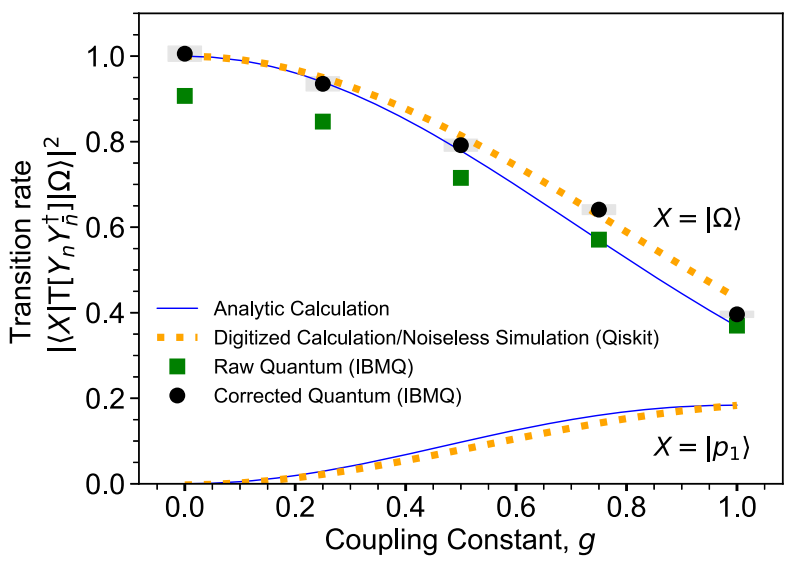

FIG. 1. Result of transition rates from the vacuum of the Wilson line for three lattice sites and $n_{Q}=2$ qubits per site to the vacuum and the lowest-lying single excited state. The solid lines show the analytical result with no field digitization while the dashed lines represent the result from a quantum simulator of our circuit. The black data points show the result from the 65-qubit IBMQ Manhattan quantum computer, corrected both for readout errors and CNOT gate errors, and the gray bands show the extrapolation errors from the extrapolation from the CNOT error correction. We only show results from the Manhattan computer for $X=\Omega$, since the circuit to measure the excited state was too deep to give reliable results.

coupling $g$ for $n_{Q}=2$ qubits per lattice site for different final states, and compare them against the analytical results, shown by black lines. Results are given for both a quantum simulation and from the 65-qubit IMBQ Manhattan quantum computer. The operators for implementing all states are exact, as the resources for doing so on a small lattice are modest. On a larger lattice approximate methods, such as KW ground state approximation and the excited state preparation techniques of [5], will be necessary; the effect of such approximations is presented in the Supplemental Material.

Errors in the quantum circuits, especially readout errors and CNOT gate errors are quite large on existing hardware. As discussed in the Supplemental Materials, the exponential of the field operator at a given position requires only $n_{Q}$ single qubit gates, such that the operator in Eq. (15) requires no CNOT gates. For $n_{Q}=2$ the state preparation requires six CNOT gates. Note that for more than three lattice sites the time evolution operator is required, which requires a much larger amount of gates, although the resulting circuits are known. For example, even for three lattice sites the standard implementation of a single Trotter step of our Hamiltonian requires 60 CNOT gates. We have applied both readout error mitigation as described in [55] as well as CNOT gate noise mitigation [56]. For more details, including Refs. [57-78], see the Supplemental Materials. One can see that the digitized result with 2 qubits per lattice site differs from the analytic calculation by up to $10 \%$. This would be reduced to at most $1.5 \%$ by adding just a single qubit per lattice site, since the resulting digitization errors fall exponentially with the number of qubits. The quantum computer reproduces the simulated result to about 5\% accuracy.

In conclusion, EFTs are well known to be able to describe the low energy dynamics of field theories and, through short distance, perturbatively computable matching coefficients, can be used to describe the dynamics of a full underlying quantum field theory. We have argued that the dynamics of a low energy EFT can be simulated with significantly smaller quantum resources than the dynamics of the corresponding full theory. In SCET the interactions of highly energetic particles with soft particles of low energy are described through operators containing Wilson lines, and we have shown in detail how the dynamics of an analogous scalar soft theory can be described using quantum algorithms. Using Wilson lines of free scalar fields in $(1+1)$ dimension, we have computed the simplest matrix elements in this soft theory, namely, the transition matrix elements from the vacuum to itself and the lowestlying excited states of two Wilson lines in opposite directions, using three lattice sites. We have compared the computations on a quantum computer to analytical results that can be obtained for this simple theory. Using only 2 qubits per lattice we obtain results within $10 \%$ of the analytical result, and by using noise-mitigation techniques, uncertainties due to running on present-day hardware can be reduced to about $5 \%$.

We would like to thank Dorota Grabowska, Bert de Jong, Michael Kreshchuk, Pier Monni, John Preskill, Martin Savage, and Miro Urbanek for useful discussions. C. W. B. and B. N. are supported by the U.S. Department of Energy (DOE), Office of Science under Contract No. DE-AC0205CH11231. In particular, support comes from Quantum Information Science Enabled Discovery (QuantISED) for High Energy Physics (KA2401032) and the Office of Advanced Scientific Computing Research (ASCR) through the Accelerated Research for Quantum Computing Program. M. F. is supported by the DOE under Grant No. DESC0010008. This research used resources of the Oak Ridge Leadership Computing Facility, which is a DOE Office of Science User Facility supported under Contract No. DEAC05-00OR22725.

*cwbauer@lbl.gov

†bpnachman@lbl.gov

*marat.freytsis@ rutgers.edu

[1] S. P. Jordan, K. S. M. Lee, and J. Preskill, Science 336, 1130 (2012).

[2] J. B. Kogut and L. Susskind, Phys. Rev. D 11, 395 (1975).

[3] J. B. Kogut, Rev. Mod. Phys. 51, 659 (1979).

[4] J. B. Bronzan, Phys. Rev. D 31, 2020 (1985).

[5] S. P. Jordan, K. S. M. Lee, and J. Preskill, Quantum Inf. Comput. 14, 1014 (2014). 
[6] A. Macridin, P. Spentzouris, J. Amundson, and R. Harnik, Phys. Rev. Lett. 121, 110504 (2018).

[7] A. Macridin, P. Spentzouris, J. Amundson, and R. Harnik, Phys. Rev. A 98, 042312 (2018).

[8] N. Klco and M. J. Savage, Phys. Rev. A 99, 052335 (2019).

[9] D. C. Hackett, K. Howe, C. Hughes, W. Jay, E. T. Neil, and J. N. Simone, Phys. Rev. A 99, 062341 (2019).

[10] K. Yeter-Aydeniz, E. F. Dumitrescu, A. J. McCaskey, R. S. Bennink, R. C. Pooser, and G. Siopsis, Phys. Rev. A 99, 032306 (2019).

[11] M. Kreshchuk, W. M. Kirby, G. Goldstein, H. Beauchemin, and P. J. Love, arXiv:2002.04016.

[12] M. Kreshchuk, S. Jia, W. M. Kirby, G. Goldstein, J. P. Vary, and P. J. Love, Entropy 23, 597 (2021).

[13] J. F. Haase, L. Dellantonio, A. Celi, D. Paulson, A. Kan, K. Jansen, and C. A. Muschik, Quantum 5, 393 (2021).

[14] D. J. Gross and F. Wilczek, Phys. Rev. Lett. 30, 1343 (1973).

[15] H. D. Politzer, Phys. Rev. Lett. 30, 1346 (1973).

[16] J. C. Collins, D. E. Soper, and G. F. Sterman, Nucl. Phys. B250, 199 (1985).

[17] S. Catani, L. Trentadue, G. Turnock, and B. R. Webber, Nucl. Phys. B407, 3 (1993).

[18] R. Bonciani, S. Catani, M. L. Mangano, and P. Nason, Phys. Lett. B 575, 268 (2003).

[19] G. Marchesini and B. R. Webber, Nucl. Phys. B238, 1 (1984).

[20] M. Bengtsson and T. Sjostrand, Nucl. Phys. B289, 810 (1987).

[21] B. Nachman, D. Provasoli, W. A. de Jong, and C. W. Bauer, Phys. Rev. Lett. 126, 062001 (2021).

[22] E. F. Dumitrescu, A. J. McCaskey, G. Hagen, G. R. Jansen, T. D. Morris, T. Papenbrock, R. C. Pooser, D. J. Dean, and P. Lougovski, Phys. Rev. Lett. 120, 210501 (2018).

[23] H. H. Lu, N. Klco, J. M. Lukens, T. D. Morris, A. Bansal, A. Ekström, G. Hagen, T. Papenbrock, A. M. Weiner, M. J. Savage, and P. Lougovski, Phys. Rev. A 100, 012320 (2019).

[24] M. J. Cervia, A. V. Patwardhan, A. B. Balantekin, S. N. Coppersmith, and C. W. Johnson, Phys. Rev. D 100, 083001 (2019).

[25] A. Roggero, A. C. Y. Li, J. Carlson, R. Gupta, and G. N. Perdue, Phys. Rev. D 101, 074038 (2020).

[26] M. J. Cervia, A. B. Balantekin, S. N. Coppersmith, C. W. Johnson, P. J. Love, C. Poole, K. Robbins, and M. Saffman, Phys. Rev. C 104, 024305 (2021).

[27] T. F. Stetina, A. Ciavarella, X. Li, and N. Wiebe, arXiv: 2101.00111.

[28] C. W. Bauer, S. Fleming, and M. E. Luke, Phys. Rev. D 63, 014006 (2000).

[29] C. W. Bauer, S. Fleming, D. Pirjol, and I. W. Stewart, Phys. Rev. D 63, 114020 (2001).

[30] C. W. Bauer and I. W. Stewart, Phys. Lett. B 516, 134 (2001).

[31] C. W. Bauer, D. Pirjol, and I. W. Stewart, Phys. Rev. D 65, 054022 (2002).

[32] C. W. Bauer, S. Fleming, D. Pirjol, I. Z. Rothstein, and I. W. Stewart, Phys. Rev. D 66, 014017 (2002).

[33] C. W. Bauer, A. V. Manohar, and M. B. Wise, Phys. Rev. Lett. 91, 122001 (2003).
[34] C. W. Bauer, C. Lee, A. V. Manohar, and M. B. Wise, Phys. Rev. D 70, 034014 (2004).

[35] A. V. Manohar, Phys. Rev. D 68, 114019 (2003).

[36] T. Becher and M. Neubert, Phys. Lett. B 637, 251 (2006).

[37] R. Goerke and M. Luke, J. High Energy Phys. 02 (2018) 147.

[38] T. Becher and M. Neubert, Phys. Lett. B 633, 739 (2006).

[39] A. H. Hoang and S. Kluth, arXiv:0806.3852.

[40] T. T. Jouttenus, I. W. Stewart, F. J. Tackmann, and W. J. Waalewijn, Phys. Rev. D 83, 114030 (2011).

[41] R. Kelley, M. D. Schwartz, R. M. Schabinger, and H. X. Zhu, Phys. Rev. D 84, 045022 (2011).

[42] P. F. Monni, T. Gehrmann, and G. Luisoni, J. High Energy Phys. 08 (2011) 010.

[43] R. Boughezal, X. Liu, and F. Petriello, Phys. Rev. D 91, 094035 (2015).

[44] I. Moult and H. X. Zhu, J. High Energy Phys. 08 (2018) 160.

[45] See Supplemental Material at http://link.aps.org/ supplemental/10.1103/PhysRevLett.127.212001 for more details on the theoretical setup and quantum circuits used.

[46] C. Lin, F. H. Zong, and D. M. Ceperley, Phys. Rev. E 64, 016702 (2001).

[47] C. T. Sachrajda and G. Villadoro, Phys. Lett. B 609, 73 (2005).

[48] P. F. Bedaque, Phys. Lett. B 593, 82 (2004).

[49] R. A. Briceno, Z. Davoudi, T. C. Luu, and M. J. Savage, Phys. Rev. D 89, 074509 (2014).

[50] H. F. Trotter, Proc. Am. Math. Soc. 10, 545 (1959).

[51] Masuo Suzuki, Commun. Math. Phys. 51, 183 (1976).

[52] Masuo Suzuki, Prog. Theor. Phys. 56, 1454 (1976).

[53] A. Kitaev and W. A. WebbarXiv:0801.0342.

[54] H. Abraham et al. https://doi.org/10.5281/zenodo.2562110.

[55] B. Nachman, M. Urbanek, W. A. de Jong, and C. W. Bauer, npj Quantum Inf. 6, 84 (2020).

[56] A. He, B. Nachman, W. A. de Jong, and C. W. Bauer, Phys. Rev. A 102, 012426 (2020).

[57] C. W. Bauer, P. Deliyannis, M. Freytsis, and B. Nachman, arXiv:2109.10918.

[58] G. D’Agostini, Nucl. Instrum. Methods Phys. Res., Sect. A 362, 487 (1995).

[59] L. B. Lucy, Astron. J. 79, 745 (1974).

[60] W. H. Richardson, J. Opt. Soc. Am. 62, 55 (1972).

[61] R. Hicks, C. W. Bauer, and B. Nachman, Phys. Rev. A 103, 022407 (2021).

[62] M. Geller and M. Sun, Quantum Sci. Technol. 6, 025009 (2021).

[63] C. Song, K. Xu, W. Liu, C.-p. Yang, S.-B. Zheng et al., Phys. Rev. Lett. 119, 180511 (2017).

[64] M. Gong, M.-C. Chen, Y. Zheng, S. Wang, C. Zha et al., Phys. Rev. Lett. 122, 110501 (2019).

[65] K. E. Hamilton et al., arXiv:2006.01805.

[66] K. X. Wei, I. Lauer, S. Srinivasan, N. Sundaresan, D. T. McClure, D. Toyli, D. C. McKay, J. M. Gambetta, and S. Sheldon, Phys. Rev. A 101, 032343 (2020).

[67] Rigetti Forest, http://docs.rigetti.com, 2020.

[68] The Cirq Contributors, https://github.com/quantumlib/Cirq, 2020. 
[69] F. Arute et al., Nat. Phys. 17, 332 (2021).

[70] The XACC Contributors, https://xacc.readthedocs.io, 2020.

[71] A. J. McCaskey et al., arXiv:1911.02452.

[72] A. J. McCaskey, Z. P. Parks, J. Jakowski, S. V. Moore, T. D. Morris, T. S. Humble, and R. C. Pooser, npj Quantum Inf. 5, 99 (2019).

[73] IBM Research, https://qiskit.org/ignis, 2019.

[74] Y. Chen, M. Farahzad, S. Yoo, and T. Wei, Phys. Rev. A 100, 052315 (2019).
[75] F. B. Maciejewski, Z. Zimboras, and M. Oszmaniec, Quantum 4, 257 (2020).

[76] E. F. Dumitrescu, A. J. McCaskey, G. Hagen, G. R. Jansen, T. D. Morris, T. Papenbrock, R. C. Pooser, D. J. Dean, and P. Lougovski, Phys. Rev. Lett. 120, 210501 (2018).

[77] S. Endo, S. C. Benjamin, and Y. Li, Phys. Rev. X 8, 031027 (2018).

[78] K. Temme, S. Bravyi, and J. M. Gambetta, Phys. Rev. Lett. 119, 180509 (2017). 